\title{
Correction to: Influence of Dietary Habits on Oxidative Stress Markers in Hashimoto's Thyroiditis by Ruggeri et al. Thyroid 2021;31:96-105. DOI: 10.1089/thy.2020.0299
}

$\mathbf{I}^{\mathrm{N}}$ N THE JANUARY 2021 issue of Thyroid (vol 31, no. 1; pp. 96-105) the article entitled "Influence of Dietary Habits on Oxidative Stress Markers in Hashimoto's Thyroiditis”' by Ruggeri et al. requires correction.

In Table 3 of the article, the values of confidence interval related to sex and body mass index were incorrectly reported, whereas odds ratio and $p$-values were correct. A footnote has also been introduced to clarify the significance.

Table 3 originally appeared as:

Table 3. Multivariate Logistic Regression Model WITH STEPWISE Procedure

\begin{tabular}{lccc}
\hline & \multicolumn{3}{c}{ Thyroid autoantibodies positivity } \\
\cline { 2 - 4 } Predictors & Odds ratio & CI & $\mathrm{p}$ \\
\hline Sex & 0.859 & $0.075-1.160$ & $\mathbf{0 . 0 0 6}$ \\
Age & 1.053 & $1.016-1.092$ & $\mathbf{0 . 0 0 5}$ \\
BMI & 0.842 & $0.759-0.958$ & 0.081 \\
PREDIMED score & 0.192 & $0.074-0.500$ & $\mathbf{0 . 0 0 1}$ \\
Meat & 2.748 & $1.721-4.387$ & $\mathbf{0 . 0 0 0 1}$ \\
Fish & 1.219 & $0.608-2.444$ & 0.577 \\
Eggs & 1.563 & $0.845-2.891$ & 0.447 \\
Dairy products & 1.462 & $1.042-2.050$ & $\mathbf{0 . 0 2 8}$ \\
Fruit and vegetables & 0.322 & $0.138-0.749$ & $\mathbf{0 . 0 0 7}$ \\
Cereals & 0.351 & $0.137-0.900$ & $\mathbf{0 . 0 2 9}$ \\
Legumes & 0.446 & $0.194-1.025$ & 0.057 \\
Olive oil & 0.455 & $0.759-7.732$ & 0.060 \\
\hline
\end{tabular}

Level of significance at $p<0.05$. Statistically significant $p$-values are in bold. The PREDIMED score was calculated as specified under the Materials and Methods section to assess adherence to the Mediterranean diet.

CI, 95\% confidence interval.
Table 3 has been revised to:

Table 3. Multivariate Logistic Regression Model WITH Stepwise Procedure ${ }^{\mathrm{a}}$

\begin{tabular}{lccc}
\hline & \multicolumn{3}{c}{ Thyroid autoantibodies positivity } \\
\cline { 2 - 4 } Predictors & Odds ratio & CI & $\mathrm{p}$ \\
\hline Sex & 0.859 & $\mathbf{0 . 7 5 9 - 0 . 9 5 8}$ & $\mathbf{0 . 0 0 6}$ \\
Age & 1.053 & $1.016-1.092$ & $\mathbf{0 . 0 0 5}$ \\
BMI & 0.842 & $\mathbf{0 . 4 9 9}-\mathbf{1 . 4 2 1}$ & 0.081 \\
PREDIMED score & 0.192 & $0.074-0.500$ & $\mathbf{0 . 0 0 1}$ \\
Meat & 2.748 & $1.721-4.387$ & $\mathbf{0 . 0 0 0 1}$ \\
Fish & 1.219 & $0.608-2.444$ & 0.577 \\
Eggs & 1.563 & $0.845-2.891$ & 0.447 \\
Dairy products & 1.462 & $1.042-2.050$ & $\mathbf{0 . 0 2 8}$ \\
Fruit and vegetables & 0.322 & $0.138-0.749$ & $\mathbf{0 . 0 0 7}$ \\
Cereals & 0.351 & $0.137-0.900$ & $\mathbf{0 . 0 2 9}$ \\
Legumes & 0.446 & $0.194-1.025$ & 0.057 \\
Olive oil & 0.455 & $0.759-7.732$ & 0.060 \\
\hline
\end{tabular}

The PREDIMED score was calculated as specified under the Materials and Methods section to assess adherence to the Mediterranean diet.

${ }^{a}$ Level of significance at $p<0.05$. Statistically significant $p$-values are in bold.

CI, $95 \%$ confidence interval.

The online version has been corrected to reflect this. The authors apologize for this error. 